Preprint typeset in JHEP style. - PAPER VERSION

CERN-TH/2000-356

FISIST/19-00/CFIF

TUM-HEP-397/00

\title{
Spontaneous CP Violation in the Next-to-Minimal Supersymmetric Standard Model Revisited
}

\author{
G.C. Branco, ${ }^{a, b}$ F. Krüger, ${ }^{c}$ J.C. Romão, ${ }^{b}$ and A.M. Teixeira ${ }^{b}$ \\ ${ }^{a}$ Theory Division, CERN, CH-1211 Geneva 23, Switzerland \\ ${ }^{b}$ Centro de Física das Interacções Fundamentais (CFIF), Departamento de \\ Física, Instituto Superior Técnico, Av. Rovisco Pais, 1049-001 Lisboa, Portugal \\ ${ }^{c}$ Physik Department, Technische Universität München, D-85748 Garching, \\ Germany \\ E-mail: gbranco@cfif.ist.utl.pt, fkrueger@ph.tum.de, \\ fromao@alfa.ist.utl.pt, ana@cfif.ist.utl.pt
}

\begin{abstract}
We re-examine spontaneous CP violation at the tree level in the context of the next-to-minimal supersymmetric standard model (NMSSM) with two Higgs doublets and a gauge singlet field. We analyse the most general Higgs potential without a discrete $Z_{3}$ symmetry, and derive an upper bound on the mass of the lightest neutral Higgs boson consistent with present experimental data. We investigate, in particular, its dependence on the admixture and CP-violating phase of the gauge singlet field, as well as on $\tan \beta$. To assess the viability of the spontaneous CP violation scenario, we estimate $\epsilon_{K}$ by applying the mass insertion approximation. We find that a non-trivial flavour structure in the soft-breaking $A$ terms is required to account for the observed $\mathrm{CP}$ violation in the neutral kaon sector. Furthermore, combining the minimisation conditions for spontaneous $\mathrm{CP}$ violation with the constraints coming from $K^{0}-\bar{K}^{0}$ mixing, we find that the upper bound on the lightest Higgs-boson mass becomes stronger. We also point out that the electric dipole moments of electron and neutron are a serious challenge for SUSY models with spontaneous CP violation.
\end{abstract}

KEywords: Spontaneous Symmetry Breaking, Supersymmetric Models, Higgs Physics, CP Violation. 


\section{Contents}

1. Introduction 1

2. The Higgs potential 3

2.1 The superpotential 3

2.2 Soft-breaking terms 3

2.3 The scalar potential and spontaneous CP violation 4

2.4 Mass spectrum 5

3. A real CKM matrix $\quad 6$

4. Content of the NMSSM $\quad 7$

4.1 Squark mass matrices 8

$\begin{array}{lll}4.2 & \text { Chargino mass matrix } & 9\end{array}$

5. Implications of indirect CP violation for the NMSSM 10

5.1 Effective Hamiltonian 10

$\begin{array}{ll}5.2 \text { Chargino box diagram } & 11\end{array}$

$\begin{array}{lll}5.3 & \text { Numerical results and discussion } & 13\end{array}$

$\begin{array}{lll}5.4 & \text { Remarks on the electric dipole moments } & 15\end{array}$

$\begin{array}{ll}\text { 6. Conclusions } & 16\end{array}$

\section{Introduction}

The origin of $\mathrm{CP}$ violation remains a fundamental open question in particle physics. In the standard model (SM), CP is explicitly broken at the Lagrangian level through complex Yukawa couplings which lead to $\mathrm{CP}$ violation in charged weak interactions via the Cabibbo-Kobayashi-Maskawa (CKM) matrix, $V_{\mathrm{CKM}}$ [1]. Although the CKM mechanism can accommodate the experimental value of $\epsilon_{K}$ (and, in principle, also $\left.\epsilon^{\prime} / \epsilon_{K}\right)$ in the neutral kaon sector, it is not clear whether it is the dominant contribution or the only one. An important motivation to consider new sources of $\mathrm{CP}$ violation stems from the fact that within the $\mathrm{SM}$ the amount of $\mathrm{CP}$ violation may not be sufficient to generate the observed baryon asymmetry in the universe [2].

An alternative scenario for the breaking of $\mathrm{CP}$ is to assume that it is a symmetry of the Lagrangian which is only spontaneously broken by the vacuum [3]. The 
purpose of the present paper is to study spontaneous breaking of CP at the tree level within the context of supersymmetry (SUSY). Although the minimal supersymmetric standard model (MSSM) has two Higgs doublets, it is well known that due to SUSY constraints on the Higgs potential it is not possible to obtain spontaneous CP violation (SCPV) at the tree level. The reason for this is entirely analogous to the situation one encounters in non-supersymmetric two-Higgs-doublet models, where SCPV cannot be achieved if a $Z_{2}$ symmetry is imposed on the Lagrangian in order to guarantee natural flavour conservation in the Higgs sector [4]. The possibility that SCPV in the MSSM might be generated through radiative corrections has been explored in Ref. [5], but this particular scenario has already been ruled out by experiment as it inevitably leads to the existence of a very light Higgs boson. Ultimately, this result is a consequence of the Georgi-Pais theorem [6]. It is therefore of interest to consider simple extensions of the MSSM such as a model with at least one gauge singlet field $(N)$ besides the two Higgs doublets $\left(H_{1,2}\right)$, the so-called next-to-minimal supersymmetric standard model (NMSSM) [7,8], and to ask if one can achieve spontaneous breaking of CP whilst generating the observed amount of $\epsilon_{K}$ and having Higgs-boson masses that are consistent with experimental data [9-13]

Whether or not the observed CP violation in the kaon sector can arise solely from supersymmetry is a serious issue. This possibility has been examined in Refs. [14-16] by simply assuming $V_{\mathrm{CKM}}$ to be real, and subsequently investigating whether SUSY sources of CP violation can account for the observed magnitude of $\epsilon_{K}$ and $\epsilon^{\prime} / \epsilon_{K}$. Here we would like to draw attention to the particularly attractive SUSY scenario with spontaneous CP symmetry breaking. The main point of this class of models is that the reality of the CKM matrix is automatic (see, e.g., Refs. [4, 17]), and no longer an ad hoc assumption. In the specific scenario we shall be considering, CP violation is caused by the phases $\phi_{D}$ and $\phi_{N}$ associated with the vacuum expectation values of $\left\langle H_{2}^{0}\right\rangle$ and $\langle N\rangle$ respectively. Being a singlet Higgs field, $N$ does not couple to quarks, and although $H_{2}^{0}$ does couple to the up-type quarks, the phase appearing in the quark mass matrix can be rotated away by redefinition of the right-handed quark fields. Consequently, this phase does not appear in the CKM matrix since the $W$-boson interactions are purely left-handed, but it does enter in other SUSY interactions.

The plan of this paper is as follows. In Sec. 2, we analyse the conditions required for the Higgs potential to have a CP-violating global minimum. We show that the upper bound on the mass of the lightest Higgs boson imposes constraints on the CP-violating phases, $\phi_{D}$ and $\phi_{N}$. Section 3 is devoted to a brief review of the real CKM matrix in supersymmetric extensions of the SM with two Higgs doublets and an arbitrary number of gauge singlet fields. In Sec. 4, we introduce the mass and mixing matrices, as well as the couplings of the Higgsino, $W$-ino, and singlet field in the NMSSM with spontaneous CP violation. The calculation of the relevant SUSY contributions to $\epsilon_{K}$ in the mass insertion approximation is presented in Sec. 5. We 
pay particular attention to the case of low $\tan \beta$ and examine the question of whether the choice of parameters of the Higgs potential that is required to obtain spontaneous $\mathrm{CP}$ violation is consistent with the experimentally observed indirect $\mathrm{CP}$ violation in the kaon sector. The implications of $\epsilon_{K}$ for the upper bound on the lightest Higgsboson mass and a new flavour structure, besides the one of the CKM matrix, are discussed. We also comment on the electric dipole moments of electron and neutron. Finally, we summarize the key features of our analysis in Sec. 6.

\section{The Higgs potential}

\subsection{The superpotential}

We are concerned with the next-to-minimal supersymmetric standard model discussed by Davies et al. [18]. The most general form of the superpotential that we analyse here is ${ }^{1}$

$$
W=W_{\text {fermion }}+W_{\text {Higgs }},
$$

with

$$
\begin{gathered}
W_{\text {fermion }}=\varepsilon_{a b}\left(h_{U}^{i j} \widehat{Q}_{i}^{a} \widehat{U}_{j} \widehat{H}_{2}^{b}+h_{D}^{i j} \widehat{Q}_{i}^{b} \widehat{D}_{j} \widehat{H}_{1}^{a}+h_{E}^{i j} \widehat{L}_{i}^{b} \widehat{R}_{j} \widehat{H}_{1}^{a}\right), \\
W_{\text {Higgs }}=-\lambda \widehat{N} \varepsilon_{a b} \widehat{H}_{1}^{a} \widehat{H}_{2}^{b}-\frac{k}{3} \widehat{N}^{3}-r \widehat{N}-\mu \varepsilon_{a b} \widehat{H}_{1}^{a} \widehat{H}_{2}^{b},
\end{gathered}
$$

where $i, j=1,2,3$ denote generation indices, $a, b=1,2$ are $\mathrm{SU}(2)$ indices, and $\varepsilon$ is a completely antisymmetric $2 \times 2$ matrix with $\varepsilon_{12}=1$. In the above expression, $\widehat{H}_{1}^{a}$ and $\widehat{H}_{2}^{a}$ denote the Higgs doublets of the minimal supersymmetric standard model and $\widehat{N}$ is a singlet field. The matrices $h_{U}, h_{D}$, and $h_{E}$ give rise to the usual Yukawa interactions which generate the masses of quarks and leptons. Since we are dealing here with spontaneous breaking of $\mathrm{CP}$ rather than explicit CP violation in the Higgs sector, these matrices are real.

\subsection{Soft-breaking terms}

In addition to the superpotential given by Eq. (2.1), we have to specify the SUSY soft-breaking terms. These are given by

$$
\mathcal{L}_{\mathrm{SB}}=\mathcal{L}_{\mathrm{SB}}^{\mathrm{fermion}}+\mathcal{L}_{\mathrm{SB}}^{\mathrm{Higgs}}
$$

where

$$
\begin{aligned}
-\mathcal{L}_{\mathrm{SB}}^{\text {fermion }} & =M_{Q_{i j}}^{2} \widetilde{Q}_{i}^{a *} \widetilde{Q}_{j}^{a}+M_{U i j}^{2} \widetilde{U}_{i} \widetilde{U}_{j}^{*}+M_{D i j}^{2} \widetilde{D}_{i} \widetilde{D}_{j}^{*}+M_{L i j}^{2} \widetilde{L}_{i}^{a *} \widetilde{L}_{j}^{a} \\
& +M_{R i j}^{2} \widetilde{R}_{i} \widetilde{R}_{j}^{*}-\frac{1}{2}\left(M_{s} \lambda_{s} \lambda_{s}+M \lambda \lambda+M^{\prime} \lambda^{\prime} \lambda^{\prime}+\text { H.c. }\right) \\
& +\varepsilon_{a b}\left(A_{U}^{i j} h_{U}^{i j} \widetilde{Q}_{i}^{a} \widetilde{U}_{j} H_{2}^{b}+A_{D}^{i j} h_{D}^{i j} \widetilde{Q}_{i}^{b} \widetilde{D}_{j} H_{1}^{a}+A_{E}^{i j} h_{E}^{i j} \widetilde{L}_{i}^{b} \widetilde{R}_{j} H_{1}^{a}\right)
\end{aligned}
$$

\footnotetext{
${ }^{1}$ This form of the superpotential has also recently been studied in Ref. [19], within the context of electroweak baryogenesis.
} 
and

$$
\begin{aligned}
-\mathcal{L}_{\mathrm{SB}}^{\mathrm{Higgs}} & =m_{H_{1}}^{2} H_{1}^{a *} H_{1}^{a}+m_{H_{2}}^{2} H_{2}^{a *} H_{2}^{a}+m_{N}^{2} N^{*} N \\
& -\left(B \mu \varepsilon_{a b} H_{1}^{a} H_{2}^{b}+A_{\lambda} N \varepsilon_{a b} H_{1}^{a} H_{2}^{b}+\frac{A_{k}}{3} N^{3}+A_{r} N+\text { H.c. }\right) .
\end{aligned}
$$

\subsection{The scalar potential and spontaneous CP violation}

Following Ref. [18], we do not require the superpotential to be invariant under a discrete $Z_{3}$ symmetry which would imply $\mu=r=0$ (thereby solving the so-called ' $\mu$ problem' of the MSSM). ${ }^{2}$ Further, we do not relate the soft SUSY-breaking parameters to some common unification scale but rather take them as arbitrary at the electroweak scale.

As it was noted by one of the authors (J.C.R.) a long time ago [12], the NMSSM with a $Z_{3}$ symmetry has no spontaneous $\mathrm{CP}$ violation (no-go theorem). The inclusion of the $Z_{3}$-breaking terms in Eq. (2.1), on the other hand, evades that no-go theorem, as was shown by Pomarol [13]. Throughout we shall assume that the treelevel potential is $\mathrm{CP}$ conserving and take all parameters real, but allow complex vacuum expectation values (VEV's) for the neutral Higgs fields which emerge after spontaneous symmetry breaking:

$$
\left\langle H_{1}^{0}\right\rangle=\frac{v_{1}}{\sqrt{2}} e^{i \theta_{1}}, \quad\left\langle H_{2}^{0}\right\rangle=\frac{v_{2}}{\sqrt{2}} e^{i \theta_{2}}, \quad\langle N\rangle=\frac{v_{3}}{\sqrt{2}} e^{i \theta_{3}} .
$$

From the superpotential and soft supersymmetry breaking terms, Eqs. (2.3) and (2.6), we derive the following CP-invariant neutral scalar potential:

$$
\begin{aligned}
V & =V_{0}+\frac{1}{8}\left(\lambda_{1} v_{1}^{4}+\lambda_{2} v_{2}^{4}\right)+\frac{1}{4}\left[\left(\lambda_{3}+\lambda_{4}\right) v_{1}^{2} v_{2}^{2}+\left(\lambda_{5} v_{1}^{2}+\lambda_{6} v_{2}^{2}\right) v_{3}^{2}\right] \\
& +\frac{1}{2} \lambda_{7} v_{1} v_{2} v_{3}^{2} \cos \left(\theta_{1}+\theta_{2}-2 \theta_{3}\right)+\frac{1}{4} \lambda_{8} v_{3}^{4}+\frac{1}{\sqrt{2}} \lambda \mu\left(v_{1}^{2}+v_{2}^{2}\right) v_{3} \cos \left(\theta_{3}\right) \\
& +\frac{1}{2}\left(m_{1}^{2} v_{1}^{2}+m_{2}^{2} v_{2}^{2}+m_{3}^{2} v_{3}^{2}\right)-\frac{1}{\sqrt{2}} m_{4} v_{1} v_{2} v_{3} \cos \left(\theta_{1}+\theta_{2}+\theta_{3}\right) \\
& -\frac{1}{3 \sqrt{2}} m_{5} v_{3}^{3} \cos \left(3 \theta_{3}\right)+m_{6}^{2} v_{1} v_{2} \cos \left(\theta_{1}+\theta_{2}\right)+m_{7}^{2} v_{3}^{2} \cos \left(2 \theta_{3}\right) \\
& +\sqrt{2} m_{8}^{3} v_{3} \cos \left(\theta_{3}\right),
\end{aligned}
$$

where $V_{0}=r^{2}$ and

$$
\begin{aligned}
& \lambda_{1}=\lambda_{2}=\frac{1}{4}\left(g^{2}+g^{2}\right), \quad \lambda_{3}=-\frac{1}{4}\left(g^{2}+g^{2}-4 \lambda^{2}\right), \quad \lambda_{4}=0, \\
& \lambda_{5}=\lambda_{6}=\lambda^{2}, \quad \lambda_{7}=\lambda k, \quad \lambda_{8}=k^{2} \\
& m_{1,2}^{2}=m_{H_{1,2}}^{2}+\mu^{2}, \quad m_{3}^{2}=m_{N}^{2}, \quad m_{4}=A_{\lambda}, \quad m_{5}=A_{k}, \\
& m_{6}^{2}=-B \mu+\lambda r, \quad m_{7}^{2}=k r, \quad m_{8}^{3}=-A_{r} .
\end{aligned}
$$

\footnotetext{
${ }^{2}$ We remind the reader that a spontaneously broken discrete symmetry may lead to cosmological domain-wall problems [20]. We do not pursue this subject here.
} 
In the numerical analysis reported here, we take the $\lambda_{l}$ 's $(l=1,2, \ldots, 8)$ as given by Eq. (2.9), i.e. in terms of $g, g^{\prime}, \lambda, k$, but consider the $m_{l}$ 's as arbitrary parameters. Referring to Eq. (2.8), we see that the doublet phases enter only in the combination $\theta_{1}+\theta_{2}$, and thus we may define

$$
\phi_{D}=\theta_{1}+\theta_{2}, \quad \phi_{N}=\theta_{3}
$$

for the doublet and singlet phase respectively. We shall henceforth set $\theta_{1}=0$ in Eq. (2.11), without loss of generality.

\subsection{Mass spectrum}

To obtain the physical masses of the NMSSM with SCPV, the following procedure has been adopted. We randomly choose sets of parameters and perform the minimisation numerically. Next, we check that the local minimum is a true one not only by verifying the minimisation conditions, but also by ensuring that all the physical Higgs bosons have positive squared masses. In calculating the neutral Higgs-boson mass matrix, radiative corrections due to top-stop loops have been included. Finally, we require that the chargino and squark masses are above the current experimental lower limits [21].

We find that an acceptable mass spectrum can be easily obtained, with the exact values depending on the set of parameters we choose. Rather than presenting tables or plots for different sets of parameters, we will concentrate on two issues: (a) the influence of the CP-violating phases on the lightest Higgs-boson mass; and (b) the maximum theoretically attainable value of its mass. As far as the former is concerned, we confirm the results of an analysis of the Higgs potential performed in Ref. [18], where it was pointed out that the large-phase solution is favoured. This feature can be clearly observed in Fig. 1, where we display the Higgs-boson mass, $m_{H^{0}}$, as a function of the phase of the gauge singlet field. It is important to note that large CP phases may be in conflict with existing limits on the electric dipole moments (EDM's) of electron and neutron. However, these constraints can be evaded, for instance, if substantial cancellations among different SUSY contributions do occur $[22,23]$ or if non-universal soft-breaking terms are present [24]. (We will return to this point in a subsequent section.)

As for the maximal possible value of the Higgs-boson mass, the result can differ from that of the MSSM for the case of large values of the coupling constant $\lambda$. In Fig. 2, we show the tree level and one-loop corrected Higgs-boson mass as a function of $\lambda$. It is apparent that only for large values of the coupling $\lambda$ the situation is different from that of the MSSM. For low values of $\lambda$, corrections to the tree level Higgs-boson mass are significant and depend mainly on the SUSY scale that we take for the squarks. This is illustrated in Fig. 3 where we plot the upper limit on $m_{H^{0}}$ versus $\tan \beta \equiv v_{2} / v_{1}$ [cf. Eq. (2.7)] for different values of $M_{\mathrm{SUSY}}$. Before leaving the 


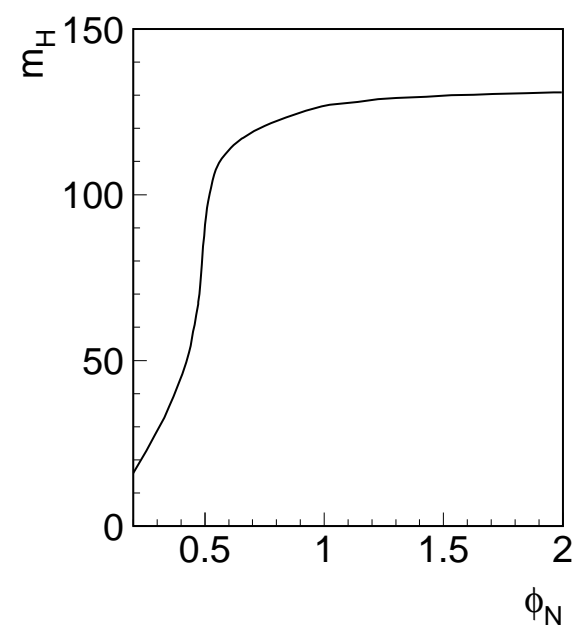

Figure 1: Maximum value of the lightest Higgs-boson mass (in $\mathrm{GeV}$ ) as a function of the CP-violating phase $\phi_{N}$ (in radians) of the Higgs singlet field.

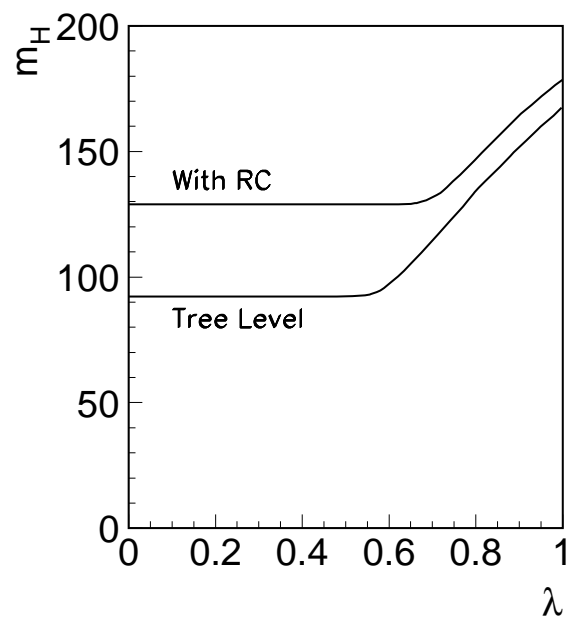

Figure 2: Maximum value of the lightest Higgs-boson mass (in $\mathrm{GeV}$ ) vs $\lambda$ at the tree level and after including radiative corrections (at one-loop level) for $M_{\mathrm{SUSY}}=1 \mathrm{TeV}$.

subject of the Higgs potential, it should be emphasized that the Higgs boson mass limits obtained at LEP for the standard model and the MSSM (see, e.g., Ref. [25]) do not necessarily apply to the NMSSM because the lightest neutral Higgs boson may have a reduced coupling to the $Z^{0}$ due to some singlet admixture $[7,26]$. In this case, the Higgs boson might not be detectable.

\section{A real CKM matrix}

We outline briefly the scenario of a real CKM matrix in the framework of supersymmetry with SCPV. In this case, CP invariance is imposed on the Lagrangian, and 


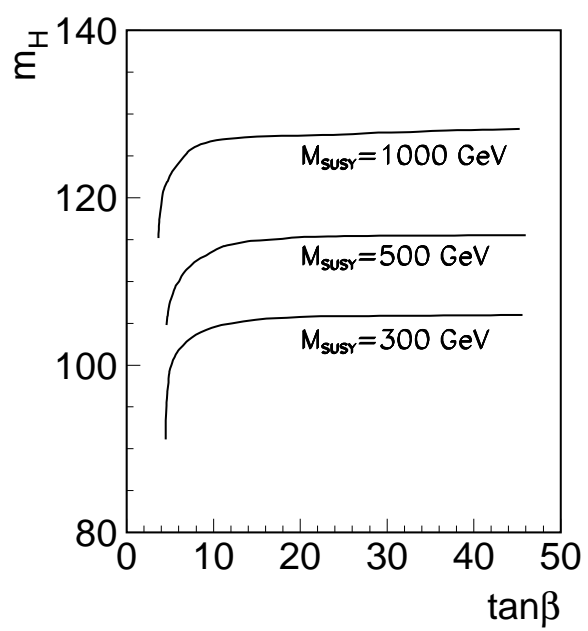

Figure 3: Upper bound on the lightest Higgs-boson mass (in $\mathrm{GeV}$ ) as a function of $\tan \beta$ for various values of $M_{\mathrm{SUSY}}$.

consequently all couplings are real. To address the question of whether the CKM mechanism occurs in the NMSSM model, let us consider the quark Yukawa couplings. As mentioned in the introduction, the gauge singlet field does not couple to quarks. Thus, the Lagrangian in terms of the weak eigenstates may be written as (omitting generation indices)

$$
\mathcal{L}_{Y}=-h_{U} H_{2}^{0 *} \bar{u}_{L} u_{R}-h_{D} H_{1}^{0 *} \bar{d}_{L} d_{R}+\text { H.c. },
$$

where $h_{U, D}$ are arbitrary real matrices in flavour space. After spontaneous symmetry breaking, the up- and down-type quarks acquire masses, namely

$$
m_{U}=h_{U} \frac{v_{2}}{\sqrt{2}} e^{-i \phi_{D}}, \quad m_{D}=h_{D} \frac{v_{1}}{\sqrt{2}} .
$$

Note that the overall phase $\phi_{D}$ can be rotated away by means of a phase transformation on $u_{R}$, i.e. $u_{R} \rightarrow u_{R}^{\prime}=e^{-i \phi_{D}} u_{R}$. Since the $W$-boson interactions are purely left-handed, this phase does not show up in the charged weak interactions. Consequently, the CKM matrix is real and CP violation arises solely from the relative phases that appear in the VEV's of the neutral Higgs fields, Eq. (2.7). An alternative way to derive the above result is to compute the weak basis invariant $T \equiv \operatorname{Tr}\left[H_{U}, H_{D}\right]^{3}$, where $H_{U} \equiv m_{U} m_{U}^{\dagger}$ and $H_{D} \equiv m_{D} m_{D}^{\dagger}$ [27]. It follows from Eq. (3.2) that $T$ vanishes, and hence there is no $\mathrm{CP}$ violation through the CKM mechanism in our model.

\section{Content of the NMSSM}

As we have argued in the previous section, all supersymmetry CP-violating phases

are equal to zero and the CKM matrix is real. Even so, the phases associated with the 
VEV's, $\phi_{D}$ and $\phi_{N}$, appear in the scalar quark, gaugino and Higgsino mass matrices, as well as in some of the vertices. We first consider the squark mass matrices.

\subsection{Squark mass matrices}

The $6 \times 6$ squark mass-squared matrices in the $\left(\tilde{q}_{L}, \tilde{q}_{R}\right)$ basis are given by

$$
M_{\tilde{q}}^{2}=\left(\begin{array}{cc}
M_{\tilde{q}_{L L}}^{2} & M_{\tilde{q}_{L R}}^{2} \\
M_{\tilde{q}_{R L}}^{2} & M_{\tilde{q}_{R R}}^{2}
\end{array}\right), \quad \tilde{q}=\tilde{U}, \tilde{D},
$$

made up of $3 \times 3$ submatrices. It proves convenient to work in the so-called 'superCKM' basis, in which the quark mass matrices are diagonal, and squarks as well as quarks are rotated simultaneously (see Ref. [28] for details). In this basis, the aforementioned submatrices are of the form

$$
\begin{aligned}
& M_{\tilde{U}_{L L}}^{2}=\left(m_{U}^{\text {diag }}\right)^{2}+V_{L}^{U} M_{\tilde{Q}}^{2} V_{L}^{U \dagger}+\frac{1}{6} M_{Z}^{2} \cos 2 \beta\left(3-4 \sin ^{2} \theta_{W}\right) \mathbb{1}, \\
& M_{\tilde{U}_{R R}}^{2}=\left(m_{U}^{\text {diag }}\right)^{2}+V_{R}^{U} M_{\tilde{U}_{R}}^{2} V_{R}^{U \dagger}+\frac{2}{3} M_{Z}^{2} \cos 2 \beta \sin ^{2} \theta_{W} \mathbb{1}, \\
& M_{\tilde{U}_{L R}}^{2}=M_{\tilde{U}_{R L}}^{2 \dagger}=V_{L}^{U} Y_{U}^{*} V_{R}^{U \dagger} \frac{v_{2}}{\sqrt{2}}-\mu_{\mathrm{eff}} \cot \beta e^{i \phi_{D}} m_{U}^{\text {diag }}, \\
& M_{\tilde{D}_{L L}}^{2}=\left(m_{D}^{\text {diag }}\right)^{2}+V_{L}^{D} M_{\tilde{Q}^{2}}^{2} V_{L}^{D \dagger}-\frac{1}{6} M_{Z}^{2} \cos 2 \beta\left(3-2 \sin ^{2} \theta_{W}\right) \mathbb{1}, \\
& M_{\tilde{D}_{R R}}^{2}=\left(m_{D}^{\text {diag }}\right)^{2}+V_{R}^{D} M_{\tilde{D}_{R}}^{2} V_{R}^{D \dagger}-\frac{1}{3} M_{Z}^{2} \cos 2 \beta \sin ^{2} \theta_{W} \mathbb{1}, \\
& M_{\tilde{D}_{L R}}^{2}=M_{\tilde{D}_{R L}}^{2 \dagger}=V_{L}^{D} Y_{D}^{*} V_{R}^{D \dagger} \frac{v_{1}}{\sqrt{2}}-\mu_{\mathrm{eff}} \tan \beta e^{i \phi_{D}} m_{D}^{\mathrm{diag}},
\end{aligned}
$$

$\theta_{W}$ being the Weinberg angle, $\mathbb{1}$ denotes a $3 \times 3$ unit matrix, and the $V$ 's diagonalize the up- and down-type quark mass matrices in Eq. (3.2). [Note that the $V$ matrices are orthogonal, and therefore $V_{\mathrm{CKM}}=V_{L}^{U}\left(V_{L}^{D}\right)^{\mathrm{T}}$.] In what follows, we take a particular case of the 'super-CKM' basis where $V_{L}^{D}=\mathbb{1}$ and $V_{L}^{U}=V_{\mathrm{CKM}}$, so that

$$
\left(m_{U}^{\text {diag }}\right)^{2}=V_{\mathrm{CKM}}\left(m_{U} m_{U}^{\dagger}\right) V_{\mathrm{CKM}}^{\mathrm{T}} .
$$

Further, we have introduced the shorthand notation

$$
\begin{aligned}
Y_{q}^{i j} \equiv A_{q}^{i j} h_{q}^{i j}, \quad q & =U, D \quad(\text { no sum over } i, j), \\
\mu_{\text {eff }} & \equiv \mu+\lambda \frac{v_{3}}{\sqrt{2}} e^{i \phi_{N}} .
\end{aligned}
$$

[Notice that Eqs. (4.2)-(4.5) show explicitly the dependence on the CP phases.] As we shall see in the next section, a non-universal flavour structure in the $A$ terms, i.e. $A_{q}^{i j} \neq$ constant, is indispensable for having sizable supersymmetry contributions to $\mathrm{CP}$ violation in the kaon sector. ${ }^{3}$ At this stage, we therefore make no assumptions regarding the nature of the several matrices and couplings involved.

\footnotetext{
${ }^{3}$ For related work, see Refs. $[14,15]$.
} 
The squark mass-squared matrix, $M_{\tilde{q}}^{2}$, can then be diagonalized by a $6 \times 6$ unitary matrix such that

$$
\left(M_{\tilde{q}}^{\mathrm{diag}}\right)^{2}=R_{\tilde{q}} M_{\tilde{q}}^{2} R_{\tilde{q}}^{\dagger}
$$

\subsection{Chargino mass matrix}

We now turn our attention to the four-component Dirac fermions, $\tilde{\chi}_{1,2}^{ \pm}$, which arise from the mixing of the $W$-inos and the charged Higgsinos. Within SUSY, the chargino mass terms in the Lagrangian are

$$
\mathcal{L}_{\text {mass }}=-\frac{1}{2}\left(\psi^{+T}, \psi^{-T}\right)\left(\begin{array}{cc}
0 & M_{\tilde{\chi}}^{T} \\
M_{\tilde{\chi}} & 0
\end{array}\right)\left(\begin{array}{c}
\psi^{+} \\
\psi^{-}
\end{array}\right)+\text {H.c. },
$$

where $\psi^{+T}=\left(-i \lambda^{+}, \tilde{H}_{2}^{+}\right), \psi^{-T}=\left(-i \lambda^{-}, \tilde{H}_{1}^{-}\right)$. The chargino mass matrix in the NMSSM with spontaneous $\mathrm{CP}$ violation reads

$$
M_{\tilde{\chi}}=\left(\begin{array}{cc}
M_{2} & \sqrt{2} M_{W} \sin \beta e^{-i \phi_{D}} \\
\sqrt{2} M_{W} \cos \beta & \mu_{\mathrm{eff}}
\end{array}\right)
$$

with the gaugino mass $M_{2}$ being real and $\mu_{\text {eff }}$ given in Eq. (4.5). As usual, $M_{\tilde{\chi}}$ can be diagonalized by means of a biunitary transformation, i.e.

$$
M_{\tilde{\chi}}^{\text {diag }}=U^{*} M_{\tilde{\chi}} V^{\dagger},
$$

where $M_{\tilde{\chi}}^{\text {diag }}$ is positive and diagonal, and $U, V$ are unitary matrices.

For convenience, we perform our calculation in the weak (rather than the physical) basis, i.e. $\tilde{W}^{-}=\left(-i \lambda^{-}, i \bar{\lambda}^{+}\right)^{\mathrm{T}}, \tilde{H}^{-}=\left(\tilde{H}_{1}^{-}, \overline{\tilde{H}}_{2}^{+}\right)^{\mathrm{T}}$, and their relation can be summarized as follows: ${ }^{4}$

$$
\begin{array}{ll}
P_{L} \tilde{W}^{-}=P_{L}\left(U_{11}^{*} \tilde{\chi}_{1}^{-}+U_{21}^{*} \tilde{\chi}_{2}^{-}\right), & P_{R} \tilde{W}^{-}=P_{R}\left(V_{11} \tilde{\chi}_{1}^{-}+V_{21} \tilde{\chi}_{2}^{-}\right), \\
P_{L} \tilde{H}^{-}=P_{L}\left(U_{12}^{*} \tilde{\chi}_{1}^{-}+U_{22}^{*} \tilde{\chi}_{2}^{-}\right), & P_{R} \tilde{H}^{-}=P_{R}\left(V_{12} \tilde{\chi}_{1}^{-}+V_{22} \tilde{\chi}_{2}^{-}\right),
\end{array}
$$

with $P_{L, R}=\left(1 \mp \gamma_{5}\right) / 2$. In order to specify the relevant couplings within the NMSSM, we recall that [29]

$$
-\mathcal{L}^{\tilde{W}, \tilde{H}}=g\left(H_{1}^{0^{*}} \tilde{\tilde{W}} P_{L} \tilde{H}+H_{2}^{0 *} \tilde{\tilde{H}} P_{L} \tilde{W}\right)+\lambda N \overline{\tilde{H}} P_{L} \tilde{H}+\text { H.c. }
$$

Then, substituting the VEV's from Eq. (2.7), and adding $W$-ino and Higgsino 'mass' terms, we arrive at

$$
-\mathcal{L}_{\text {int }}=m_{\tilde{W}} \overline{\tilde{W}} \tilde{W}+m_{\tilde{H}} \overline{\tilde{H}} \tilde{H}+\frac{g}{\sqrt{2}}\left(v_{1} e^{-i \varphi} \overline{\tilde{W}}_{R} \tilde{H}_{L}+v_{2} e^{i \phi_{D}} \overline{\tilde{W}}_{L} \tilde{H}_{R}+\text { H.c. }\right),
$$

\footnotetext{
${ }^{4}$ We define $\tilde{W}^{-}$and $\tilde{H}^{-}$as particles, contrary to Ref. [29].
} 
where

$$
m_{\tilde{W}}=M_{2}, \quad m_{\tilde{H}}=\left|\mu_{\mathrm{eff}}\right|, \quad \varphi=\arg \left(\mu_{\mathrm{eff}}\right),
$$

and $\mu_{\text {eff }}$ as in Eq. (4.5). In deriving Eq. (4.12), we have adjusted our definition of the phase of the left-handed Higgsino field such that $\tilde{H}_{L} \rightarrow e^{-i \varphi} \tilde{H}_{L}$. As for gluino and neutralino interactions, we will argue in the next section that to good approximation the chargino box diagram may be regarded as the dominant contribution to $\epsilon_{K}$ (see also Refs. [11, 13]).

\section{Implications of indirect CP violation for the NMSSM}

In this section, we wish to explore the consequences of SCPV for the upper bound of the lightest Higgs-boson mass derived in Sec. 2 by taking into account CP violation in $K^{0}-\bar{K}^{0}$ mixing. To accomplish this, we will compute the box-diagram contributions to $\epsilon_{K}$ by applying the mass insertion approximation [30]. That is, adopting the notation

$$
M_{\tilde{q}}^{2} \equiv\left(M_{\tilde{q}}^{0}\right)^{2}+M_{\tilde{q}}^{\prime 2}, \quad\left(M_{\tilde{q}}^{0}\right)^{2}=\operatorname{diag}\left(a_{1}^{0}, \ldots, a_{6}^{0}\right),
$$

and recalling Eq. (4.6), we may write to first order in $M_{\tilde{q}}^{\prime 2}$ [31]

$$
\left(R_{\tilde{q}}^{\dagger}\right)_{m k} f\left(a_{k}\right)\left(R_{\tilde{q}}\right)_{k n}=\delta_{m n} f\left(a_{n}^{0}\right)+\left(M_{\tilde{q}}^{\prime 2}\right)_{m n} F\left(a_{m}^{0}, a_{n}^{0}\right)
$$

$f$ being an arbitrary function, $k=1, \ldots, 6$, and

$$
F(x, y)=\frac{f(x)-f(y)}{x-y}
$$

\subsection{Effective Hamiltonian}

Let us start with the effective Hamiltonian governing $\Delta S=2$ transitions, which can be written in the form [32]:

$$
H_{\mathrm{eff}}=\sum_{i} c_{i} \mathcal{O}_{i}
$$

where the $c_{i}$ 's are the Wilson coefficients describing the short-distance interactions while $\mathcal{O}_{i}$ denote local operators which may be found, e.g., in Refs. [33,34]. The off-diagonal element of the neutral kaon mass matrix is related to $H_{\text {eff }}$ through

$$
\mathcal{M}_{12}=\frac{\left\langle K^{0}\left|H_{\mathrm{eff}}\right| \bar{K}^{0}\right\rangle}{2 m_{K}}
$$

and its imaginary part gives rise to (assuming $\epsilon^{\prime} \ll \epsilon_{K}$ )

$$
\epsilon_{K} \simeq \frac{e^{i \pi / 4}}{\sqrt{2}} \frac{\operatorname{Im} \mathcal{M}_{12}}{\Delta m_{K}}
$$




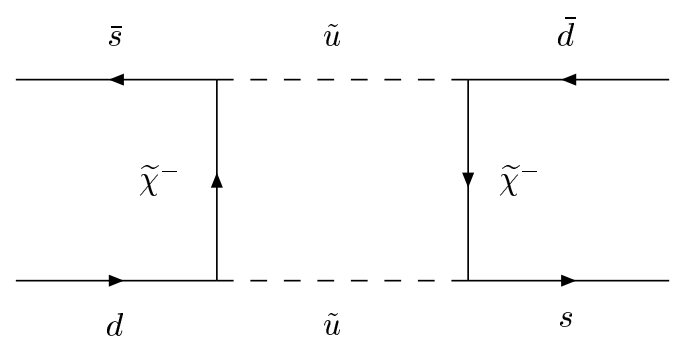

Figure 4: The dominant box-diagram contribution to the off-diagonal element $\operatorname{Im} \mathcal{M}_{12}$ in the neutral kaon mass matrix within the framework of the NMSSM.

with the experimental values of $\Delta m_{K}=(3.489 \pm 0.008) \times 10^{-12} \mathrm{MeV}^{5}$ and $\left|\epsilon_{K}\right|=$ $(2.271 \pm 0.017) \times 10^{-3}[21]$.

The Wilson coefficients $c_{i}$ in the presence of SUSY contributions can be decomposed as follows:

$$
c_{i}=c_{i}^{W}+c_{i}^{H^{ \pm}}+c_{i}^{\tilde{\chi}^{ \pm}}+c_{i}^{\tilde{g}}+c_{i}^{\tilde{\chi}^{0}} .
$$

Regarding the various contributions in Eq. (5.7), we confine ourselves to a relatively few remarks. First, there are no $W$-boson and charged Higgs-boson contributions to the imaginary part of $\mathcal{M}_{12}$ since the CKM matrix is real. Second, in subsequent calculations we will choose a basis where the down-type Yukawa matrices are diagonal. As a consequence, the flavour off-diagonal gluino contributions are either zero or real in the approximation of retaining only a single mass insertion in an internal squark line. Third, box diagrams with neutralinos are expected to compete with the chargino contributions only for large values of $\tan \beta$ (i.e. $\tan \beta \sim 50$ ), and hence are less important for the present scenario with low $\tan \beta$. In the remainder of this section, we will therefore focus on the chargino box diagram with scalar up-type quarks (see Fig. 4) as it gives by far the dominant contribution in our model.

Turning to the operators $\mathcal{O}_{i}$ in Eq. (5.4), the $\Delta S=2$ transition within the model under study is largely governed by the $V-A$ four-fermion operator $\mathcal{O}_{1}=$ $\bar{d} \gamma^{\mu} P_{L} s \bar{d} \gamma_{\mu} P_{L} s$. As a matter of fact, the new-physics contribution to the remaining operators (i.e. scalar and pseudoscalar operators) are suppressed relative to the chargino contribution either through small quark masses, $m_{s, d}$, or small Yukawa couplings. Thus, it is fairly reasonable to consider only the non-standard contribution to the Wilson coefficient $c_{1}$. The relevant hadronic matrix element in the vacuum saturation approximation is given by $\left\langle K^{0}\left|\mathcal{O}_{1}\right| \bar{K}^{0}\right\rangle=(2 / 3) f_{K}^{2} m_{K}^{2}$, with $f_{K}=160 \mathrm{MeV}$.

\subsection{Chargino box diagram}

In the $\tilde{W}-\tilde{H}$ basis, and making use of the mass insertion approximation, the chargino

\footnotetext{
${ }^{5}$ Note that for $\Delta m_{K}$, and following standard procedure, we have used the experimental value. No useful bound on the present model can be derived from $\left.\Delta m_{K}\right|_{\text {exp }}$, since it receives important long-distance contributions.
} 

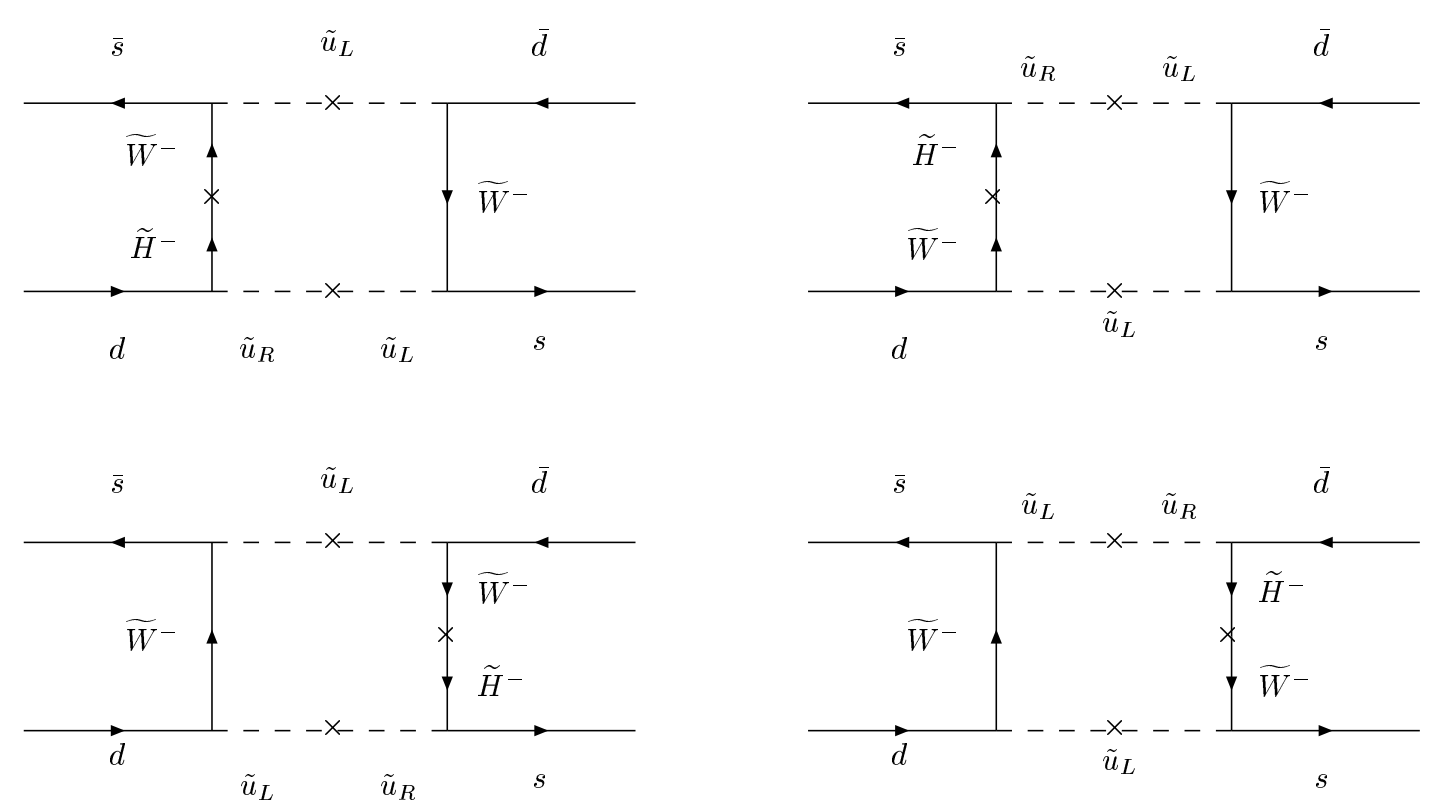

Figure 5: The main contributions to $\epsilon_{K}$ in the mass insertion approximation with $W$-ino and Higgsino exchange.

contributions to the short-distance coefficient $c_{1}$ can be written as a sum of 16 individual terms, each corresponding to a particular box diagram. Introducing the notation $\{i j\}(i, j=L, R)$ for the various mass insertions, these contributions can be classified in terms of the nature of the mass insertion in the internal squark line and the number of $\tilde{W}-\tilde{H}$ exchanges in the sfermion line. That is, the relevant amplitude for $c_{1}$ can be symbolically written as

$$
\begin{aligned}
A_{c_{1}}^{\tilde{\chi}^{ \pm}} & \sim\left(X_{\tilde{W}-\tilde{H}}\right)^{0}[\{L L\}\{L L\}+\{R R\}\{R R\}+\{L R\}\{R L\}] \\
& +\left(X_{\tilde{W}-\tilde{H}}\right)^{1}[\{L L\}\{L R\}+\{L R\}\{R R\}] \\
& +\left(X_{\tilde{W}-\tilde{H}}\right)^{2}[\{L L\}\{R R\}+\{L R\}\{L R\}] .
\end{aligned}
$$

Only one diagram belongs to the $\{L L\}\{L L\}$ and $\{R R\}\{R R\}$ mass insertions, whereas we find two (four) diagrams associated with the $\{L R\}\{R L\},\{L L\}\{R R\},\{L R\}\{L R\}$ $(\{L L\}\{L R\},\{L R\}\{R R\})$ mass insertions. It turns out that the dominant contribution to $c_{1}$ corresponds to the class of diagrams with $\left(X_{\tilde{W}-\tilde{H}}\right)^{1}\{L L\}\{L R\}$, depicted in Fig. 5, in agreement with the findings of Refs. [11,13]. The calculation of the dominant contributions to $\operatorname{Im} \mathcal{M}_{12}$ for the case of non-degenerate left-handed upsquarks leads to a cumbersome expression. Hence, for the sake of simplicity, we perform the calculation in the limit of degenerate left-handed up-type squarks, and find that the expression for $\operatorname{Im} \mathcal{M}_{12}$ becomes much simpler, and its physical interpretation more transparent. (We have numerically verified that small values of the off-diagonal elements of $M_{\tilde{Q}}^{2}$ have little effect on the $\tilde{u}_{L}$ mass splitting.) In that 
limit, i.e. $m_{\tilde{u}_{L}}=\left\langle m_{\tilde{q}}\right\rangle$, where $\left\langle m_{\tilde{q}}\right\rangle$ represents the average squark mass of the first two generations, we obtain

$$
\begin{aligned}
& \operatorname{Im} \mathcal{M}_{12}=\frac{2 G_{F}^{2} f_{K}^{2} m_{K} m_{W}^{4}}{3 \pi^{2}\left\langle m_{\tilde{q}}\right\rangle^{8}}\left(V_{t d}^{*} V_{t s}\right) m_{t}^{2}\left|e^{i \phi_{D}} m_{\tilde{W}}+\cot \beta m_{\tilde{H}}\right| \\
& \times\left\{\Delta A_{U} \sin \left(\varphi_{\chi}-\phi_{D}\right)\left(M_{\tilde{Q}}^{2}\right)_{12} I\left(r_{\tilde{W}}, r_{\tilde{H}}, r_{\tilde{u}_{L}}, r_{\tilde{t}_{R}}\right)\right\}
\end{aligned}
$$

with $m_{\tilde{H}, \tilde{W}}$ and $\left(M_{\tilde{Q}}^{2}\right)$ as defined in Eqs. (4.13) and (4.2) respectively. Here we have used unitarity of the CKM matrix and kept only the leading top-quark contribution. In the above formula, $r_{i}=m_{i}^{2} /\left\langle m_{\tilde{q}}\right\rangle^{2}, \Delta A_{U} \equiv A_{U}^{13}-A_{U}^{23}$, and

$$
\varphi_{\chi}=\arg \left(e^{i \phi_{D}} m_{\tilde{W}}+\cot \beta m_{\tilde{H}}\right) \text {. }
$$

The function $I$ in Eq. (5.9) can be reduced successively to elementary functions for appropriate limits of its arguments (see Refs. [23,31]). For ease of writing, we give the result for the special case

$$
I(x, x, 1,1)=\frac{1}{2}\left[\frac{10+19 x+x^{2}}{3(1-x)^{5}}+\frac{\left(1+6 x+3 x^{2}\right)}{(1-x)^{6}} \ln x\right],
$$

which corresponds to the scenario with $m_{\tilde{H}}=m_{\tilde{W}}$ and degenerate squarks. The appearance of $A_{U}^{i 3}(i=1,2)$ terms in Eq. (5.9) is closely related to having the Higgsino coupling to down and strange quarks in distinct diagrams, and stems from the trilinear soft-breaking terms in $Y_{U}^{i j} \equiv A_{U}^{i j} h_{U}^{i j}$ [Eq. (4.4)]. From inspection of Eq. (5.9), it is straightforward to conclude that in order to get $\operatorname{Im} \mathcal{M}_{12}$ different from zero we need to move aside from a theory of universal $A_{U}$ terms (i.e. $\Delta A_{U} \neq 0$ ) in other words, it is not possible to saturate the observed CP violation in the $K$ meson system in the context of SUSY with a real CKM matrix and universal $A_{U}$ terms $[14,15] .{ }^{6}$ Let us note parenthetically that the need for a special $A_{U}^{i 3}(i=1,2)$ texture as a key element to get the experimentally measured $\epsilon_{K}$ has also been pointed out in Ref. [14], although in a different scenario.

Since the sign of $\Delta A_{U}$ is not fixed, we do not include the constraint $\operatorname{Re} \epsilon_{K}>0$ in the analysis which follows.

\subsection{Numerical results and discussion}

For our numerical calculations, we have used the nominal values

$$
\left(M_{\tilde{Q}}^{2}\right)_{12} /\left\langle m_{\tilde{q}}\right\rangle^{2}=0.08, \quad V_{t d}=0.0066, \quad V_{t s}=-0.04, \quad m_{t}=175 \mathrm{GeV} .
$$

Notice that the value of $V_{t d}$ differs from the one determined in the context of the SM with CP violation by fitting to experimental data; this is due to the fact that we are

\footnotetext{
${ }^{6}$ It has recently been pointed out in Ref. [35] that there might be a connection between a nontrivial flavour structure and sizable SUSY CP violation, thereby avoiding domain-wall problems.
} 
Table 1: Numerical values of $\left|\epsilon_{K}\right|$ in the low $\tan \beta$ region for certain sets of model parameters that satisfy the minimisation condition of the Higgs potential. We have chosen $\Delta A_{U}=500 \mathrm{GeV}$ for the non-universal $A$ terms, as described in the text.

\begin{tabular}{ccccccccc}
\hline \hline $\begin{array}{c}\left|\epsilon_{K}\right| \\
\left(10^{-3}\right)\end{array}$ & $\begin{array}{c}\phi_{D} \\
(\mathrm{rad})\end{array}$ & $\begin{array}{c}\phi_{N} \\
(\mathrm{rad})\end{array}$ & $\begin{array}{c}m_{H^{0}} \\
(\mathrm{GeV})\end{array}$ & $\begin{array}{c}\left\langle m_{\tilde{q}}\right\rangle \\
(\mathrm{GeV})\end{array}$ & $\begin{array}{c}m_{\tilde{t}_{R}} \\
(\mathrm{GeV})\end{array}$ & $\tan \beta$ & $\lambda$ & $\begin{array}{c}v_{3} \\
(\mathrm{GeV})\end{array}$ \\
\hline 3.24 & 4.71 & 1.57 & 99 & 252 & 235 & 6.7 & -0.03 & 327 \\
3.03 & 0.89 & 1.75 & 97 & 261 & 168 & 6.6 & +0.33 & 387 \\
2.75 & 4.71 & 4.71 & 99 & 232 & 201 & 9.2 & -0.02 & 221 \\
2.42 & 1.96 & 4.08 & 94 & 299 & 174 & 5.1 & -0.06 & 352 \\
2.10 & 4.67 & 4.75 & 98 & 279 & 220 & 7.8 & +0.01 & 142 \\
2.02 & 4.68 & 4.71 & 92 & 250 & 152 & 7.4 & +0.02 & 371 \\
2.01 & 4.18 & 4.73 & 96 & 280 & 232 & 4.6 & -0.01 & 238 \\
1.31 & 1.12 & 4.72 & 100 & 273 & 241 & 9.6 & -0.01 & 238 \\
1.29 & 2.35 & 4.70 & 99 & 258 & 230 & 6.1 & -0.13 & 363 \\
\hline \hline
\end{tabular}

dealing with a flat triangle and the corresponding orthogonality constraints. ${ }^{7}$ The remaining parameters are evaluated numerically by minimising the Higgs potential (see Sec. 2). Our results for the absolute value of $\epsilon_{K}$ for various sets of SUSY parameters and low $\tan \beta$ are reported in Table 1 . Recall that the numerical values we present for $\left|\epsilon_{K}\right|$ have been derived for degenerate left-handed squarks. Taking into account corrections due to non-degeneracy will lead to a very small enhancement, typically a few percent, for $\operatorname{Im} \mathcal{M}_{12}$. Hence, the values for $\left|\epsilon_{K}\right|$ listed in Table 1 may be interpreted as a lower bound. As far as $M_{2}$ and $\mu$ are concerned, we obtain the ranges $90 \mathrm{GeV} \lesssim M_{2} \lesssim 160 \mathrm{GeV}$ and $120 \mathrm{GeV} \lesssim|\mu| \lesssim 270 \mathrm{GeV}$. Turning to the non-universal flavour structure, it is obvious from Eq. (5.9) that there is a linear dependence of $\epsilon_{K}$ on the relative difference $\Delta A_{U}$. In order to saturate the value of $\left|\epsilon_{K}\right|$ and to obey present experimental limits on the sparticle spectrum, one has to take $\Delta A_{U}$ of order $500 \mathrm{GeV}$. A detailed discussion of scenarios where such values for $A_{U}^{i 3}(i=1,2)$ appear lies beyond the scope of this paper. We will just mention that values around the $\mathrm{TeV}$ scale do not significantly affect the mass spectrum of the theory, and that they can account for values of the left-right mass insertion, i.e.

$$
\left(\delta_{L R}^{U}\right)_{i 3}=\frac{v_{2}}{\sqrt{2}} \frac{\left(V_{L}^{U} Y_{U}^{*} V_{R}^{U \dagger}\right)_{i 3}}{\left\langle m_{\tilde{q}}\right\rangle^{2}},
$$

as small as 0.02 and 0.08 for $\left(\delta_{L R}^{U}\right)_{13}$ and $\left(\delta_{L R}^{U}\right)_{23}$ respectively, which are consistent

\footnotetext{
${ }^{7}$ The value used for $\left|V_{t d}\right|$ is consistent with the experimental value of $B_{d}^{0}-\bar{B}_{d}^{0}$ mixing, provided one assumes a new-physics contribution to $\Delta m_{B_{d}}$ of at least $20 \%$. To be specific, we have chosen a positive value for $\rho$. As for a negative value of $\rho$, orthogonality of the CKM matrix would imply $\left|V_{t d}\right|=0.011$.
} 
with the bounds coming from the measurement of $b \rightarrow s \gamma$ branching fraction. (Note that these $\delta$ 's also affect the decay $b \rightarrow s l^{+} l^{-}$.) Indeed, according to Ref. [28], $\left(\delta_{L R}^{U}\right)_{23}$ is constrained to be $\left|\left(\delta_{L R}^{U}\right)_{23}\right| \lesssim 3\left(\left\langle m_{\tilde{q}}\right\rangle / 500 \mathrm{GeV}\right)^{2}$, which is only useful if $\left\langle m_{\tilde{q}}\right\rangle \lesssim 300 \mathrm{GeV}$. Similarly, the chargino contribution to $B_{d}^{0}-\bar{B}_{d}^{0}$ mixing leads to the constraint $\left(\delta_{L R}^{U}\right)_{13} \lesssim 0.1\left(\left\langle m_{\tilde{q}}\right\rangle / 500 \mathrm{GeV}\right)[31] .^{8}$

At this point, a few remarks are in order regarding previous studies contained in the literature. As we have already mentioned, the diagrams depicted in Fig. 5 give the leading contributions to $\operatorname{Im} \mathcal{M}_{12}$, in agreement with Refs. [11,13]. The final form of our result, however, differs from that of previous works in several ways. First, we have used the mass insertion approximation to compute the dominant contributions to $\epsilon_{K}$, which enables us to use other largely model-independent constraints on the off-diagonal elements of the squark mass matrices. Second, we have considered a nontrivial structure for the $L R$ squark mass-squared terms, which proved to be crucial in order to have indirect $\mathrm{CP}$ violation within the present model. More importantly, our parameter space is severely constrained since in the calculation of the various contributions to $\epsilon_{K}$ we have considered only those sets of parameters that correspond to true minima of the Higgs potential.

We conclude this section with several comments on large CP phases and their implications for the EDM's of electron and neutron.

\subsection{Remarks on the electric dipole moments}

As seen from Table 1, the CP phase of the gauge singlet field, $\phi_{N}$, is accompanied by small values of $\lambda$ so that the EDM constraints are less stringent [37]. Further, the SUSY contributions to the EDM's depend only on flavour-diagonal entries in the squark mass matrix, whereas the expression for $\epsilon_{K}$ involves flavour off-diagonal elements. However, it is evident from Eq. (4.2) that there is an overall CP-violating phase which is independent of the new flavour structure. Apart from the requirement that $\left.\left.A_{U}^{i j}\right|_{i=j} \ll A_{U}^{i j}\right|_{i \neq j}$, a conceivable way to suppress the EDM's includes large cancellations between different SUSY contributions and a SUSY particle spectrum with masses of scalar quarks and gauginos in the TeV range. Given the analytic results for the contributions to the EDM's of electron and neutron mediated by photino and gluino [33], together with the sets of parameters displayed in Table 1 and the present experimental results of $d_{n}<6.3 \times 10^{-26} e \mathrm{~cm}$ (90\% C.L.) and $d_{e}=1.8 \times 10^{-27} e \mathrm{~cm}[21]$, the photino and gluino masses are required to satisfy $0.5 \mathrm{TeV} \lesssim m_{\tilde{\gamma}} \lesssim 2 \mathrm{TeV}$ and $2 \mathrm{TeV} \lesssim m_{\tilde{g}} \lesssim 6 \mathrm{TeV} .^{9}$

Such a hierarchy in the soft gaugino masses requires further discussion. We first note that masses of the superpartners of about $1 \mathrm{TeV}$ may be in conflict with the

\footnotetext{
${ }^{8}$ Note that the requirement of vacuum stability also leads to upper bounds on $\left(\delta_{L R}^{U}\right)_{i 3}(i=1,2)$ [36]. The nominal values used in our numerical analysis are compatible with these upper limits.

${ }^{9}$ For simplicity, we have assumed $m_{\tilde{l}}=m_{\tilde{q}}$. Note that the constraint on $m_{\tilde{g}}$ is relaxed if we allow for an order-of-magnitude variation in the value of the hadronic matrix elements.
} 
cosmological relic density. In addition, such a scale for the $\tilde{\gamma}$ and the $\tilde{g}$ masses is rather unnatural, since the masses of the squarks and $W$-ino are typically of the order $100-300 \mathrm{GeV}$ in the model under consideration. [Similar conclusions can be drawn if we take into account the chargino and neutralino contributions (see, e.g., Ref. [23]).] Finally, we stress that the above-mentioned hierarchy for the scalar partners leads to an unacceptable scenario for the lightest supersymmetric particle (LSP). In this case, the LSP would be either charged (one of the lightest squarks or a chargino), or would have a non-zero lepton number. To summarize, it appears that unless the parameters of the model are fine-tuned, there will be large contributions to the EDM's of electron and neutron, in potential conflict with experiment. ${ }^{10}$

\section{Conclusions}

The origin of $\mathrm{CP}$ violation, in particular, the question of whether $\mathrm{CP}$ is explicitly or spontaneously broken, is an important issue in particle physics. In this paper, we have studied spontaneous CP violation in the context of the NMSSM, demonstrating that it is possible to generate sufficient $\mathrm{CP}$ violation in order to account for the magnitude of $\epsilon_{K}$. We have emphasized that the NMSSM with spontaneous CP violation is a natural framework to discuss the possibility of having CP violation that arises entirely from SUSY interactions. This is due to the fact that in the above-mentioned scenario the CKM matrix is automatically real.

By performing a complete and systematic study of the Higgs potential in the NMSSM with spontaneous CP violation, we have shown that the minimisation of the most general Higgs potential leads to an acceptable mass spectrum which is accompanied by large CP-violating phases. We have argued that the lightest neutral Higgs boson can have a reduced coupling to the $Z^{0}$ due to the additional singlet field, and thus may escape detection at LEP. We have shown that it is possible to have a mass of the lightest neutral Higgs boson of about $125 \mathrm{GeV}$. However, such values for the Higgs-boson mass require a relatively large value for $M_{\mathrm{SUSY}}$ of the order $O(1 \mathrm{TeV})$. By contrast, a rather low SUSY scale with $M_{\text {SUSY }} \approx 300 \mathrm{GeV}$ (i.e. light squark and $W$-ino masses) and a non-trivial flavour structure of the soft SUSY-breaking trilinear couplings $A_{U}^{i 3}(i=1,2)$ are required in order to account for the observed CP violation in $K^{0}-\bar{K}^{0}$ mixing. As a consequence, the parameter space is severely constrained and the mass of the lightest Higgs boson is further diminished, and it turns out to be no greater than $\sim 100 \mathrm{GeV}$ for the case of low $\tan \beta(\lesssim 10)$. As far as large CP phases are concerned, we have argued that it may be difficult to reconcile the large-phase solution with the severe constraints on the EDM's of electron and neutron. Although we do not exclude the possibility of cancellations between different contributions, we

\footnotetext{
${ }^{10}$ As far as the $B$ system is concerned, we merely remark that the model can accommodate a large CP asymmetry $a_{J / \psi}$, i.e. a large value of $\sin 2 \beta$ (see, e.g., Ref. [10]).
} 
find this highly unlikely in view of the constrained parameter space. Therefore, the implications of the EDM bounds on the parameter space, as well as the implied LSP scenario, are a great challenge for SUSY models with spontaneous CP violation (at least within the minimal model we have considered here).

\section{Acknowledgments}

One of us (A.M.T.) would like to thank Ricardo González Felipe for useful discussions, and the Theory Division at CERN for its kind hospitality during the final stage of this work. This project was supported in part by the TMR Network of the EC under contract ERBFMRX-CT96-0090. A.M.T. acknowledges support by 'Fundação para a Ciência e Tecnologia' under grant PRAXIS XXI BD/11030/97.

\section{References}

[1] N. Cabibbo, Phys. Rev. Lett. 10 (1963) 531; M. Kobayashi and K. Maskawa, Prog. Theor. Phys. 49 (1973) 652.

[2] A.G. Cohen, D.B. Kaplan, and A.E. Nelson, Annu. Rev. Nucl. Part. Sci. 43 (1993) 27; M.B. Gavela, P. Hernandez, J. Orloff, O. Pène, and C. Quimbay, Nucl. Phys. B430 (1994) 345; ibid. B430 (1994) 382; A.D. Dolgov, hep-ph/9707419; V.A. Rubakov and M.E. Shaposhnikov, Usp. Fiz. Nauk 166 (1996) 493 [Phys. Usp. 39 (1996) 461].

[3] T.D. Lee, Phys. Rev. D8 (1973) 1226.

[4] G.C. Branco, Phys. Rev. D22 (1980) 2901; Phys. Rev. Lett. 44 (1980) 504.

[5] N. Maekawa, Phys. Lett. B282 (1992) 387; A. Pomarol, ibid. B287 (1992) 331; N. Haba, ibid. B398 (1997) 305; O.C.W. Kong and F.L. Lin, ibid. B419 (1998) 217; O. Lebedev, Eur. Phys. J. C4 (1998) 363.

[6] H. Georgi and A. Pais, Phys. Rev. D10 (1974) 1246.

[7] J. Ellis, J.F. Gunion, H.E. Haber, L. Roszkowski, and F. Zwirner, Phys. Rev. D39 (1989) 844; S.F. King and P.L. White, ibid. D52 (1995) 4183; ibid. D53 (1996) 4049.

[8] M. Drees, Int. J. Mod. Phys. A4 (1989) 3635; U. Ellwanger, M. Rausch de Traubenberg, and C.A. Savoy, Z. Phys. C67 (1995) 665; Nucl. Phys. B492 (1997) 21.

[9] K.S. Babu and S.M. Barr, Phys. Rev. D49 (1994) R2156; N. Haba, M. Matsuda, and M. Tanimoto, ibid. D54 (1996) 6928; S.W. Ham, S.K. Oh, and H.S. Song, Phys. Rev. D61 (2000) 055010.

[10] O. Lebedev, Int. J. Mod. Phys. A15 (2000) 2987.

[11] O. Lebedev, Phys. Lett. B452 (1999) 294. 
[12] J.C. Romão, Phys. Lett. B173 (1986) 309.

[13] A. Pomarol, Phys. Rev. D47 (1993) 273.

[14] S.A. Abel and J.-M. Frère, Phys. Rev. D55 (1997) 1623.

[15] S. Khalil, T. Kobayashi, and A. Masiero, Phys. Rev. D60 (1999) 075003; D.A. Demir, A. Masiero, and O. Vives, ibid. D61 (2000) 075009.

[16] S. Baek, J.-H. Jang, P. Ko, and J.H. Park, Phys. Rev. D62 (2000) 117701; M. Brhlik, L. Everett, G.L. Kane, S.F. King, and O. Lebedev, Phys. Rev. Lett. 84 (2000) 3041.

[17] G.C. Branco, F. Cagarrinho, and F. Krüger, Phys. Lett. B459 (1999) 224.

[18] A.T. Davies, C.D. Froggatt, and R.G. Moorhouse, Phys. Lett. B372 (1996) 88; A.T. Davies, C.D. Froggatt, and A. Usai, Proceedings of the International Europhysics Conference on High-Energy Physics, edited by D. Lellouch et al. (Springer-Verlag, Berlin, 1999), p. 891, hep-ph/9712501; Proceedings of Strong and Electroweak Matter '98, edited by J. Ambjoern et al. (World Scientific, Singapore, 1999), p. 227, hep$\mathrm{ph} / 9902476$.

[19] S.J. Huber and M.G. Schmidt, hep-ph/0003122.

[20] Ya.B. Zel'dovich, I.Yu. Kobzarev, and L.B. Okun, Zh. Eksp. Teor. Fiz. 67 (1974) 3 [Sov. Phys. JETP 40 (1974) 1]; S.A. Abel, S. Sarkar, and P.L. White, Nucl. Phys. B454 (1995) 663; S.A. Abel, ibid. B480 (1996) 55; C. Panagiotakopoulos and K. Tamvakis, Phys. Lett. B469 (1999) 145.

[21] Particle Data Group, D.E. Groom et al., Eur. Phys. J. C15 (2000) 1.

[22] T. Ibrahim and P. Nath, Phys. Rev. D58 (1998) 111301; ibid. D60 (1999) 099902 (E); M. Brhlik, G.J. Good, and G.L. Kane, ibid. D59 (1999) 115004; A. Bartl, T. Gajdosik, W. Porod, P. Stockinger, and H. Stremnitzer, ibid. D60 (1999) 073003.

[23] S. Pokorski, J. Rosiek, and C.A. Savoy, Nucl. Phys. B570 (2000) 81.

[24] See, for example, S. Khalil, T. Kobayashi, and O. Vives, Nucl. Phys. B580 (2000) 275.

[25] M. Bastero-Gil, C. Hugonie, S.F. King, D.P. Roy, and S. Vempati, Phys. Lett. B489 (2000) 359; G.L. Kane, S.F. King, and Lian-Tao Wang, hep-ph/0010312, and references therein.

[26] U. Ellwanger, M. Rausch de Traubenberg, and C.A. Savoy, Phys. Lett. B315 (1993) 331; J.F. Gunion, H.E. Haber, and T. Moroi, hep-ph/9610337; B. Grzadkowski, J.F. Gunion, and J. Kalinowski, Phys. Rev. D60 (1999) 075011; U. Ellwanger and C. Hugonie, Eur. Phys. J. C (to be published), hep-ph/9909260.

[27] J. Bernabéu, G.C. Branco, and M. Gronau, Phys. Lett. 169B (1986) 243. 
[28] M. Misiak, S. Pokorski, and J. Rosiek, in Heavy Flavours II, edited by A.J. Buras and M. Lindner (World Scientific, Singapore, 1998), p. 795, hep-ph/9703442.

[29] J.F. Gunion, H.E. Haber, G.L. Kane, and S. Dawson, The Higgs Hunter's Guide (Addison-Wesley, Reading, MA, 1990); hep-ph/9302272 (E); H.E. Haber and G.L. Kane, Phys. Rep. 117 (1985) 75; J.F. Gunion and H.E. Haber, Nucl. Phys. B272 (1986) 1; ibid. B402 (1993) 567 (E).

[30] L.J. Hall, V.A. Kostelecky, and S. Raby, Nucl. Phys. B267 (1986) 415; see also M. Dugan, B. Grinstein, and L. Hall, Nucl. Phys. B255 (1985) 413.

[31] A.J. Buras, A. Romanino, and L. Silvestrini, Nucl. Phys. B520 (1998) 3.

[32] See, for example, G. Buchalla, A.J. Buras, and M.E. Lautenbacher, Rev. Mod. Phys. 68 (1996) 1125.

[33] F. Gabbiani, E. Gabrielli, A. Masiero, and L. Silvestrini, Nucl. Phys. B477 (1996) 321.

[34] M. Ciuchini et al., JHEP 10 (1998) 008; A.J. Buras, M. Misiak, and J. Urban, Nucl. Phys. B586 (2000) 397.

[35] D.A. Demir, Phys. Rev. D62 (2000) 075003.

[36] J.A. Casas and S. Dimopoulos, Phys. Lett. B387 (1996) 107.

[37] M. Matsuda and M. Tanimoto, Phys. Rev. D52 (1995) 3100. 